\title{
Increment thresholds against pulsed and steady backgrounds
}

\author{
LEONARD MATIN and ALAN KORNHEISER \\ Columbia University, New York, New York 10027
}

\begin{abstract}
Cornsweet and Pinsker reported that $\Delta \mathrm{L} / \mathrm{L}$ was constant at all values of $\mathrm{L}$ for briefly pulsed fields ( $\Delta \mathrm{L}$ was the luminance increment required for discriminating which of two nearby flashed fields was brighter). We obtain the same result when the subject must detect which of two briefly (4-msec) flashed backgrounds contains an increment at its center. However, when the background and increment are flashed at a longer duration (200 $\mathrm{msec}) \Delta \mathrm{L} / \mathrm{L}$ decreases with increasing $\mathrm{L}$ as is obtained with a steady background. Two previous models which handle earlier work do not readily accommodate this result; we suggest a theoretical approach that does.
\end{abstract}

In 1965, Cornsweet and Pinsker described increment threshold experiments in which two nearby fields were simultaneously flashed for identical brief durations. One of the fields was set at the base or background luminance, $\mathrm{L}$, and the second contained a luminance increment added to $\mathrm{L}$. The threshold luminance increment $\Delta \mathrm{L}$ required for discriminating which of the two fields was brighter was determined over a large range of $L$. They reported that $\Delta \mathrm{L} / \mathrm{L}$ does not monotonically decrease toward an asymptote with increase in $\mathrm{L}$, as it does in the classical data for luminance discrimination obtained with test flashes presented against a steady background. Instead, for all background luminances above absolute threshold, $\Delta \mathrm{L} / \mathrm{L}$ remained constant at about the same value that was obtained with steady backgrounds of high luminance.

Their explanation of the divergence of results contained two main features: (1) The nervous system performs two main operations in the process of extracting the ratio $\Delta \mathrm{L} / \mathrm{L}$ : an early stage yields a neural response that is proportional to the logarithm of the stimulus. This transformation is done in spatially separate channels for each of the two stimuli. The linear difference between the neural responses to $\mathrm{L}$ and to $\mathrm{L}+\Delta \mathrm{L}$ is obtained from a later stage as the output of a synapse into which both nerve impulse packages are sent-synapses being essentially linear transducers. A particular value of such a difference from this later stage is assumed to be the threshold value for discrimination, and such a constant difference will be obtained from the model for a constant value of $\Delta \mathrm{L} / \mathrm{L}$ regardless of the value of $L$. (2) They also employ the concept of a "time window" within which nerve impulses from the early stage are counted; they point out

This research was supported by PHS Grant EY 00375 from the National Eye Institute, National Institute of Health, and by Research Grant NSF-BMS73-01463 from the National Science Foundation. that the constant difference in response will hold across luminance levels if the "time window" is either constant over all levels of luminance, or-if it does vary-remains longer than the duration of the responses to the two stimuli being compared. This time window is then assumed to be equivalent to a critical duration, and the critical neural response to a stimulus-incremental test flash, continuous background, or flashed background-is the neural output of the early stage integrated over the length of one critical duration. Thus, this integrated output to a given stimulus will be independent of variation in the value of critical duration as long as critical duration remains longer than the duration of response. However, for a stimulus whose total response is longer than critical duration, only the segment of the response equal in length to critical duration is integrated within a particular time window (see Figure 1). Clearly then-within this theoretical framework-if both the background luminance and luminance increment are sufficiently brief and of the same constant duration, background luminance can be changed without concern that any consequent changes in critical duration will result in changes in $\Delta \mathrm{L} / \mathrm{L} .{ }^{1}$ However, when threshold $\Delta \mathrm{L}$ for a brief constant duration flash is measured against a steady background, variations in critical duration will influence the integrated response to the steady background (see Figure 2), but no influence on the response to the test flash from an early or later stage will result even if critical duration does change with background luminance; as critical duration decreases, then, the integrated neural response to the steady background will suffer, while that to the test flash will not, and if the constant difference in final neural response is to be maintained, threshold $\Delta \mathrm{L}$ must decrease with decreasing critical duration.

It can be shown that the above reasoning leads simply to $\tau=t_{2} \cdot\left(\Delta \mathrm{L}_{1} / \Delta \mathrm{L}_{2}\right)$ where $\tau$ is critical duration, $t_{2}$ is test flash duration, and $\Delta L_{1}$ and 


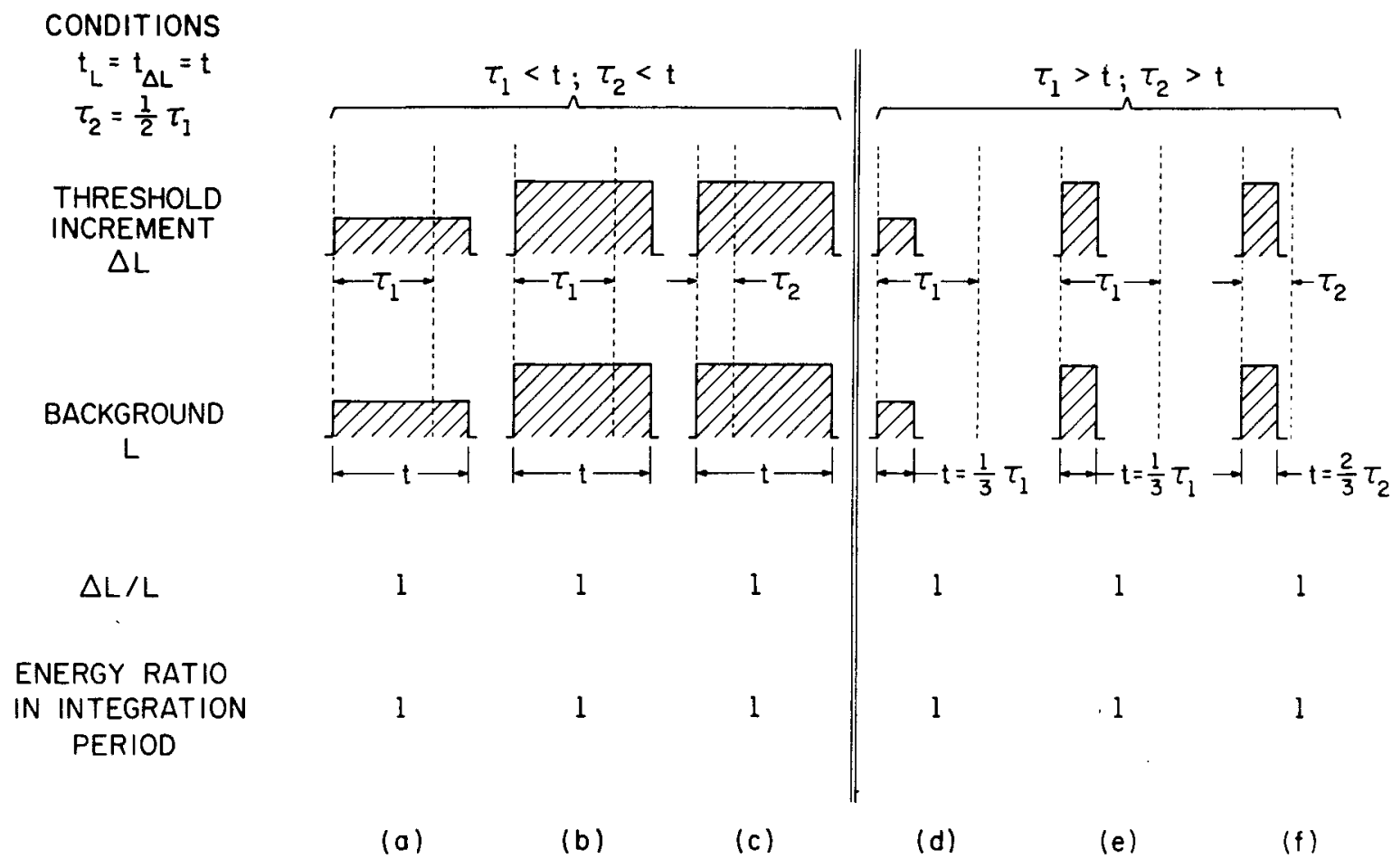

Figure 1. This figure represents relations between the "time window" and duration and luminance of adaptation and test stimuli that are involved in increment threshold discriminations for flashed backgrounds as these relations derive from Cornsweet and Pinsker's model. It shows that with flash duration equal and synchronous for test and background stimuli, the prediction for threshold $\Delta L / L$ is that it remains constant if the duration of the "time window" is longer than flash duration (a-c) or if "time window" is shorter than flash duration (d-f). In each panel, the height'of the stimulus is proportional to the luminance, $L$ or $\Delta L$, the width is proportional to the duration, $t$, the dashed lines delimit a duration equal to the time window, the indicated ratio of stimulus energy is the ratio of the energy in the increment to the energy in the background integrated over the time window. The ratios shown all yield the same predicted difference in neural response and all are at threshold if any one of them is. In panel $b$, the background luminance is twice the value in (a), and the time window $\left(\tau_{1}\right)$ is the same as in (a). Consequently, the test flash luminance, $\Delta \mathrm{L}$, needs to be doubled to obtain the same criterion difference in neural response on Cornsweet and Pinsker's theory. In (a), neural response is proportional to log $\Delta L-$ log $L$, or $\log \Delta \mathrm{L} / \mathrm{L}$; in (b), neural response is proportional to $\log 2 \Delta \mathrm{L}-\log 2 \mathrm{~L}$, or $\log \Delta \mathrm{L} / \mathrm{L}$. In (c), the background luminance is the same as in (b), but the critical duration is assumed to have been halved; the difference in neural response remains unchanged. Similar reasoning hold for panels $d-f$.

$\Delta \mathrm{L}_{2}$ are the threshold luminance increments against a steady background and flashed background, respectively. It also can be shown that this theory in effect reduces to a single requirement under all conditions: regardless of the value of the luminance of the background, the value of the time window width, the relation of the durations of time window and stimuli, or whether the background is flashed or steady, it is required that the ratio of stimulus energy in the increment to that in the background-each quantity integrated over a single time window width-be constant in order to obtain a constant response out of the later stage of the model (see Figures 1 and 2).

Cornsweet and Pinsker's theory of the increment threshold is of particular interest in relation to another one (Matin, 1968), in which it was proposed that the time constant of a neural network is modified by the level of adapting luminance and that this modification yields linked changes in gain ${ }^{2}$ and in critical duration; the change in gain influences the response to stimuli of short and of long duration.
Cornsweet and Pinsker's theory treats changes in duration of the time window and in gain as if they were the result of separate mechanisms which process the response in successive stages. Thus, in the latter theory, changes in gain arise in an early stage as a result of the setting of base luminance, $L$, which determines the segment of the logarithmic stimulusresponse operating characteristic at which the incremental stimulus taps in (e.g., the response to $L_{2}$ is smaller when there is a stimulus $L_{1}$ in the same channel than when there is none: see Matin and Kornheiser, 1976, Figure 1b). It is only in a subsequent stage that the time window limits the portion of the response that is "counted"; hence, gain for longer stimuli may be changed, leaving the gain for brief stimuli unaffected.

Cornsweet and Pinsker's (1965) finding of $\Delta L / L$ constancy was obtained with 4.5-msec flashes of test and background, a duration below any reasonable expectations for critical duration. (In fact, their predicted values of critical duration are as low as $8 \mathrm{msec}$ at high luminances.) However, not only does 
their theory predict $\Delta \mathrm{L} / \mathrm{L}$ constancy for the case in which test and background are flashed sufficiently briefly so that-regardless of any variation in critical duration with variation in $\mathrm{L}$-both remain below critical duration if all other things remain equal, but it also predicts constancy with increments and background that are both long relative to critical duration, a consequence to which they did not refer. For this condition, the integrated response to the test flash and to the background should suffer equally when critical duration is reduced, leaving $\Delta \mathrm{L} / \mathrm{L}$ unchanged for variations in critical duration with $\mathrm{L}$.

But when long duration flashes are presented against steady backgrounds both flash and background are available for longer than critical duration, and for this condition, $\Delta \mathrm{L} / \mathrm{L}$ decreases monotonically with L (cf. Barlow, 1958; Graham \& Kemp, 1938). Clearly, then, either other factors mediate the difference in results between short and long flashes or the Cornsweet and Pinsker formulation does not hold at all; in any case, it cannot be sufficient as an account of the main mechanism underlying the increment threshold under all conditions. Among those factors that are likely to produce differences in the processing of flashes of short and long duration are those connected with the effects of involuntary eye movements on the processing of visual information at the border between the test flash and background and at the border between the background and its surround. ${ }^{3}$ The influences that might be introduced by these movements are complicated. However, if a differential influence of eye movements with $\mathrm{L}$ were to occur, it would, if anything, be expected to raise $\Delta \mathrm{L} / \mathrm{L}$ at higher values of $\mathrm{L}-\mathrm{a}$ prediction that does not hold. In any case, influence of eye movements on responses to flashes cannot be the main basis for variation of $\Delta \mathrm{L} / \mathrm{L}$ with steady backgrounds, since this variation is identical with short- as well as long-duration flashes within critical duration.

There is a basic plausibility about some aspects of the mechanism proposed by Cornsweet and Pinsker which suggests that further exploration is desirable. In addition, there remains the constancy

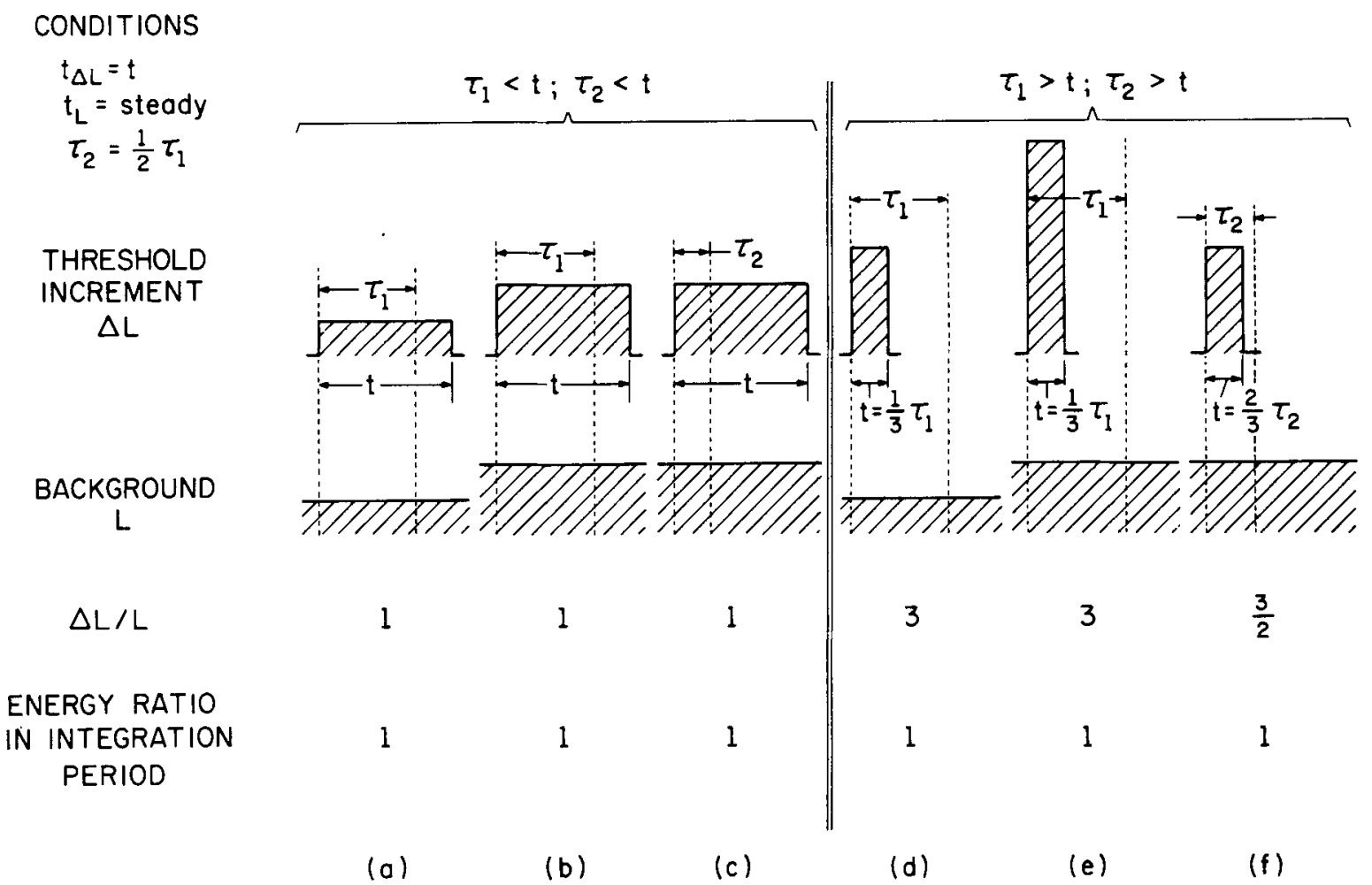

Figure 2. This figure carries out the same didactic operations for Cornsweet and Pinsker's model of increment threshold for steady backgrounds that Figure 1 does for flashed backgrounds. It shows that when the constant duration test flash is longer than the time window (a-c), the predicted threshold $\Delta \mathrm{L} / \mathrm{L}$ does not change with decrease in width of the time window, but that when the constantduration test flash is shorter than the time window (d-f), $\Delta \mathrm{L} / \mathrm{L}$ is predicted to decrease with decrease in width of the tine window. In (e), it is assumed that, although the steady background luminance is twice that in (d), the width of the time window has not changed; thus $\Delta L / L$ is the same in (d) and (e). However, if the width of the time window does change, as in (f), then so does threshold incremental luminance-and so $\Delta \mathrm{L} / \mathrm{L}$-in order to maintain the same difference in neural response from the later stage of the Cornsweet and Pinsker model as holds in all other panels of Figures 1 and 2. Therefore, it should be clear that two separable factors are controlling the threshold value of $\Delta \mathrm{L}$ : the magnitude of the background luminance and the width of the time window. In Figures 1 and 2 , these two factors are assumed to be separable for didactic purposes. However, Cornsweet and Pinsker find that the window width required to treat their data by their theory decreases with increase of the steady background luminance; they assume that window width is constant with luminance variation of the flashed background. 
of $\Delta \mathrm{L} / \mathrm{L}$ for brief flashes to be explained on other grounds if the theory is not valid. The present experiment was intended to provide information that would relate to a decision between alternative mechanisms for producing changes in gain although it was not construed as a critical test. In addition, we were interested in determining whether Cornsweet and Pinsker's results with a 4.5-msec flashed background could be obtained with a background of longer duration.

The essential point in the present experiment involves flashing both test flash and background simultaneously, but at a duration longer than critical duration. If $\Delta \mathrm{L} / \mathrm{L}$ varies with $\mathrm{L}$ here, it would appear, at least on the surface, that it cannot be a consequence of a change in gain due to a change of the constant relating response and the logarithm of the stimulus since both increment and background are transients of the same duration; some departure from logarithmicity would seem to be required as a modification if the Cornsweet and Pinsker theory is to be maintained at all. On the other hand, constancy of $\Delta \mathrm{L} / \mathrm{L}$ with $\mathrm{L}$ here would be consistent with the idea that some difference in gain for flashes and steady background was the basis for the results with steady background and long flashes.

In addition, several other elements were built into the experiment: (1) Since the earlier result with steady background (Graham \& Kemp, 1936) was obtained with a yes-no task, and since Cornsweet and Pinsker suggest the possibility that some important aspects of their data depended on the use of a spatial forced-choice procedure, we employed a spatial forced-choice procedure in the conditions with steady background as well as in the conditions with flashed background. (2) In addition, since they added a luminance increment to one of the two entire background targets on each trial, the task was essentially a spatial discrimination between two nearby brightnesses when the two targets $(L, L+\Delta L)$ were flashed (i.e., which is brighter?). However, when the backgrounds were steady, it was only necessary to detect which target changed relative to its own immediately previous state. Since this difference may have been involved in the difference in the two outcomes, we performed our experiments with smalldiameter luminance increment targets on large backgrounds; thus, for both flashed and steady backgrounds, the task was the same: it required the subject to discriminate which background contained the small target. Neither condition required (any more than the other condition) that the two targets be compared to each other.

\section{APPARATUS AND PROCEDURE}

Three beams of a four-beam Maxwellian-view optical system were employed. The system was modified relative to previous descriptions (Matin, 1962; Stewart, 1972) by combining the two main beams for each eye into a four-beam monocular system. It employed glow modulator lamps as light sources and was powered by an interface that itself was driven by a Linc- 8 computer. The optical unit sat outside of a light-tight booth located in a larger room, and was arranged so that the subject could be seated comfortably inside the booth with his head position determined by a fitted mouthbite that itself was attached to the wall of the booth which the subject faced. Protruding through this wall at eye level was the front of the optical unit whose main portion rested outside on top of a base that extended as the lower half of the wall and into which the subject's legs protruded.

The view presented to the subject (see Figure $3 a$ ) contained a continuously present circular fixation target $1 \mathrm{~min}$ in diameter at a luminance of $1 \mathrm{log}$ trol. Two circular background fields of 25.0-min diameter were horizontally separated from the fixation target by $22.5 \mathrm{~min}$, one on each side of the fixation target. Both background fields were from a single beam, and when they were presented were always presented together and at the same luminance. The third beam contained the two test targets, $5.0 \mathrm{~min}$ in diameter, each centered on one of the two background fields; only one of the test targets was ever presented on a given trial.

The experiment involved four conditions with background luminance as a parameter of each condition. In two of the conditions, the two background fields were continuously present throughout the series of trials along with the fixation target (Figure 3, Conditions A and B). On each trial, the test target centered on one of the two backgrounds was flashed, the other test target being blocked. In one of these two "steady background" conditions, the test flash was presented for $4 \mathrm{msec}$; in the second condition, the test flash was presented for $200 \mathrm{msec}$. In the other two conditions, only the fixation target was present between trials. On each trial, both background fields were flashed along with one or the other test target, all presented simultaneously for the same duration (Figure 3, Conditions $C$ and D). This duration was $4 \mathrm{msec}$ in one of these "flashed-background" conditions and $200 \mathrm{msec}$ in the other condition. Our choice of the number of background luminances employed and their values was determined by our attempt at comparing the four conditions; we did not attempt to obtain complete and detailed $\Delta \mathrm{L} / \mathrm{L}$ vs. L functions.

The full experiment took 13 sessions for each of the two subjects. Condition $C$ (Figure 3 ) was run only on the first 2 days of the experiment, Condition $A$ on the next 4 days, Condition $D$ on
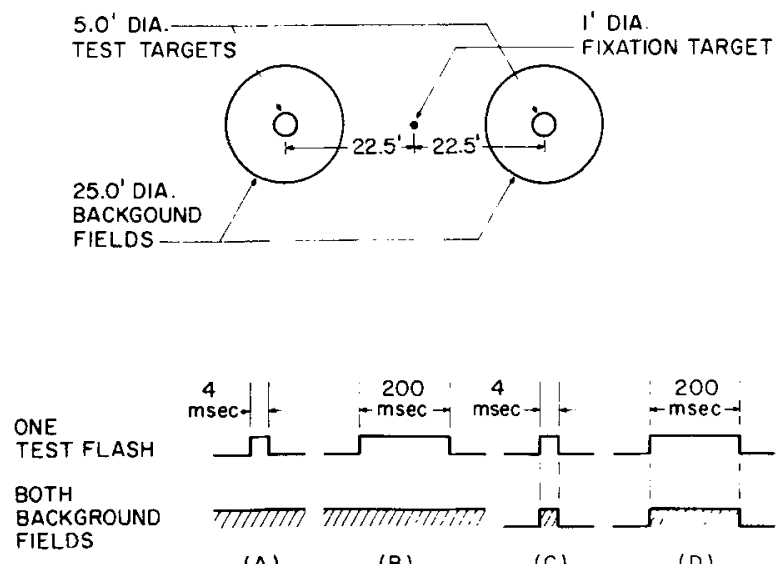

(A)

(B)

(C)

(D)

Figure 3. The spatial configuration of background, test targets, and fixation target is shown in the top panel. The temporal pattern for each of the four conditions is shown below: both background fields were steady in Conditions $A$ and $B$; they were both flashed synchronously with one of the test targets on each trial of Conditions $C$ and $D$. 
the next 3 days, and Condition $B$ on the last 4 days. Prior to each session, the subject was dark-adapted for $15 \mathrm{~min}$. This was followed by 2 min of fixation and light adaptation to the steady background at the luminance level that was to be tested only when one of the steady background conditions was to follow. Otherwise, following dark adaptation and 10 warm-up trials with test flashes at the $90 \%$ level, the data collection began. Within a session, data were collected at two levels of background luminance, and all measurements were made at one of the two levels before the second was employed. A given luminance level for a given condition was employed in two sessions, being tested in the first half of the session in one of the two sessions and in the second in the other. Testing at a given luminance level within each session involved presentation of 80 trials in eight blocks of 10 trials each. Each block involved presentation of a fixed level for the increment flash with presentation on 5 trials each on the left and 5 on the right background; the order within the 10 trials was random. Four luminance levels within the uncertainty range were employed for the test flash, and all four were run through once (the four levels were randomly ordered within the four blocks) before any one was tested again. Thus each level of increment flash was presented on a total of 40 trials over the two sessions in which the condition was run; on 20 trials, it was superimposed on the left background, and on the other 20 , on the right background.

On each trial, the subject initiated the flash by pressing a pushbutton switch with his left hand. He then reported whether the test flash appeared on the left or the right background, and was immediately told if he had been correct.

\section{RESULTS AND DISCUSSION}

$\log \Delta \mathrm{L} / \mathrm{L}$ is plotted against $\log \mathrm{L}$ in Figure 4 for subject L.M. for Conditions A-D. The results for subject J.M. were similar. Each of the $\Delta \mathrm{L}$ values is the increment in retinal illuminance that was reported correctly on $75 \%$ of the trials for that condition; these values were obtained from probit fits to the psychometric functions. ${ }^{4}$

The major point to be noted about these data is that, for the case in which both background and test fields were simultaneously flashed for $200 \mathrm{msec}$. (Condition D), we obtained a monotonic decrease in $\Delta \mathrm{L} / \mathrm{L}$ with increase in $\mathrm{L}$ such as characterized the results with the steady background (Conditions $A$ and $B$ ), although constancy of $\Delta \mathrm{L} / \mathrm{L}$ with luminance was obtained when the fields were flashed for $4 \mathrm{msec}$. Thus the constancy of $\Delta \mathrm{L} / \mathrm{L}$ reported by Cornsweet and Pinsker for a flashed-background condition with brief flash durations is not a general property of flashed-background conditions; it does not hold when a long flash duration is employed. As indicated in the introduction above, these results argue against Cornsweet and:Pinsker's "time window" theory.

Since the results for the 4-msec flashed background condition do not extend to the low values of $\log \mathrm{L}$ at which the knees of the curve for the other conditions were obtained, one might question whether a knee and rise in $\Delta \mathrm{L} / \mathrm{L}$ might not be found for the 4-msec flashed background condition also if background luminance had been extended to lower values. However, the lowest value of background luminance employed for the $4 \mathrm{msec}$ flashed background condition was very dim and lay only about $.6 \mathrm{log}$ units

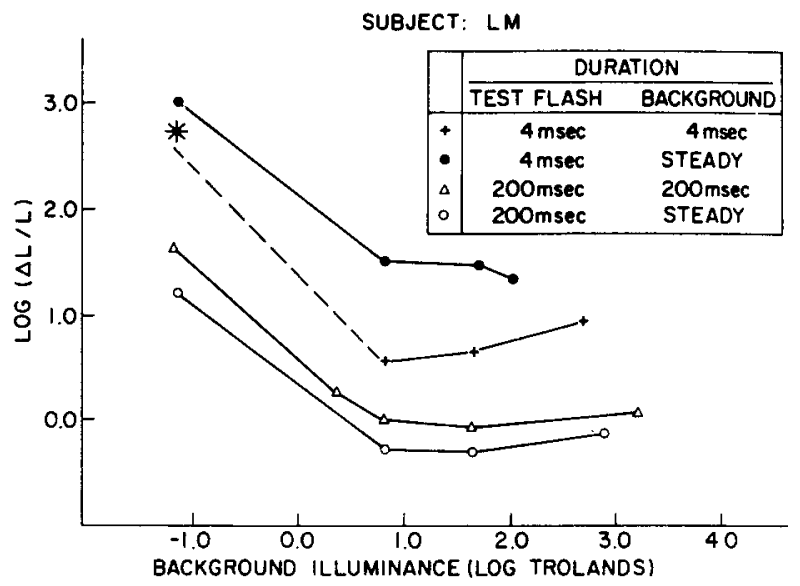

Figure 4. $\log \Delta \mathrm{L} / \mathrm{L}$ plotted against background retinal illuminance for each of the four conditions of the present experiment. The dashed line has a slope of -1.0 ; absolute threshold for the 4-msec flashed background was about .6 log unit below the point at which the dashed line intersects the data. The asterisk is the logarithm of the absolute threshold for the 4-msec test flash. It lies above the dashed line by about $.15 \mathrm{log}$ unit, indicating that it was higher by this amount than the value of $\Delta \mathrm{L}$ in Condition $\mathrm{C}$ against a background of $0.84 \mathrm{log}$ units of illuminance (see Footnote 3).

above the absolute threshold for 4-msec flashes of the background. Further reduction in luminance of the 4-msec background would not be expected to yield any further reduction in $\Delta \mathrm{L}$. [Indeed, when a measurement of $\Delta \mathrm{L}$ against an unilluminated background was made, it was slightly higher (.1-.2 log units) than against the dimmest background employed in condition C. ${ }^{3,6}$ ] Thus, while it is true that once $\Delta \mathrm{L}$ had reached a minimum further reduction in $\mathrm{L}$ would have caused $\Delta \mathrm{L} / \mathrm{L}$ to increase, such an increase would be most reasonably treated as an artifact of calculation and therefore is shown in Figure 4 as a dashed line. In the other three conditions $(A, B, D)$, there is no question but that the background is clearly visible at the lowest luminance levels employed for these conditions.

It will now be useful to reexamine Cornsweet and Pinsker's (1965) results. We have reproduced the relevant portion of their data in Figure $5 a$ as they originally presented it. It is important to note that what is plotted is $\Delta L / L$ vs. $\log L-\log L_{o}$, where $\mathrm{L}_{\mathrm{o}}$ represents absolute threshold for the background. But the background was steady under one condition and flashed for $4.5 \mathrm{msec}$ under the other condition. Therefore, $\mathrm{L}_{\mathrm{o}}$ was approximately $1.8 \mathrm{log}$ units lower for the steady background condition than for the flashed-background condition, and the curve for the flashed-background condition was shifted $1.8 \mathrm{log}$ units to the left of its true position on a scale that plots the logarithm of stimulus luminance. In Figure $5 \mathrm{~b}$, we have replotted the data from Figure 5a on a true luminance scale such as the scale in Figure 4 on which our data were plotted. It is clear that when this is done, the large difference between 

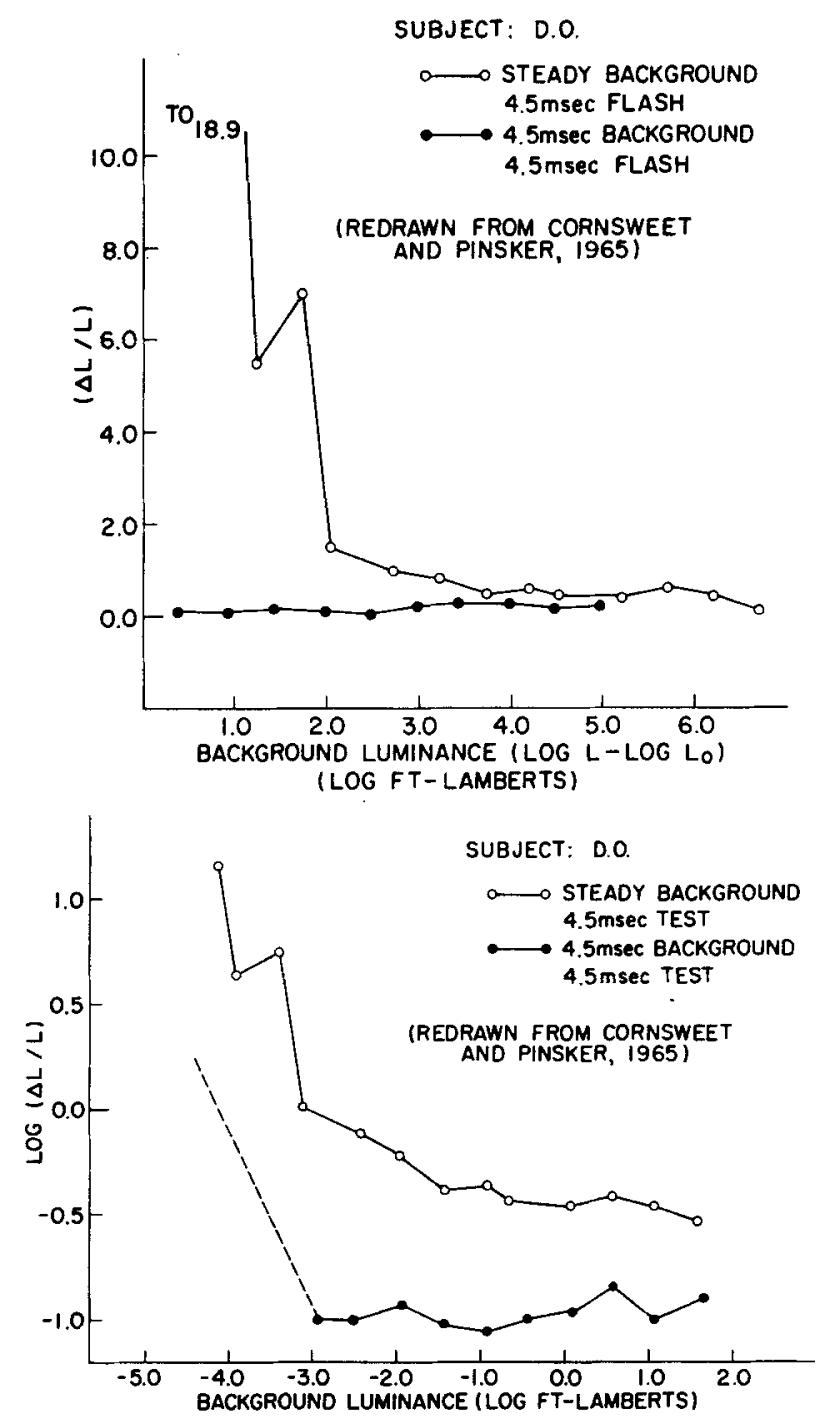

Figure 5. (a) Reproduction of graph from Cornsweet and Pinsker (1965) for the two conditions relevant to the present report; these are similar to our Conditions $A$ and $C$. (b) The data in (a) are replotted with the abscissa in units of background luminance instead of units of background luminance above threshold. The dashed line has a slope of -1 .

flashed and steady background conditions which appears in Figure 5a at low luminances is greatly diminished. Indeed, as an examination of Figure $5 b$ will reveal, for the range of intensities at which the pulsed background remains visible, there exists a range of background luminances of at most $1 \mathrm{log}$ unit for which the shape of the functions significantly differ; our less detailed data (Figure 4) contain no range at all over which the shape of the functions differs. The dashed line in Figure 5b shows (as in Figure 4) what would be expected if $\Delta \mathrm{L}$ did not change with further reductions in $L$ into the region where the flashed $L$ is invisible (slope of -1 ). As our earlier comments indicate, Cornsweet and Pinsker's decision not to show how $\Delta \mathrm{L} / \mathrm{L}$ varies in the region where the flashed background cannot be seen is a reasonable one. However, it is worth noting that the background becomes invisible for 4-msec flashes at a point that happens to be close to the knee of the $\Delta \mathrm{L} / \mathrm{L}$ function, and that, although we have not carried out a detailed investigation of this point, the location of this knee seems determined primarily by background luminance. Since visibility of the background is determined by both luminance and duration, long-duration backgrounds will fail to show $\Delta \mathrm{L} / \mathrm{L}$ constancy (as in our $200-\mathrm{msec}$ flashed background condition) while short duration backgrounds will exhibit $\Delta \mathrm{L} / \mathrm{L}$ constancy-but the latter holds only as long as the decision is followed not to plot $\Delta \mathrm{L} / \mathrm{L}$ where $\mathrm{L}$ is not visible. In any case, the "time window" theory does not appear capable of handling our data for test flashes of long duration against either pulsed or steady backgrounds.

Although Cornsweet and Pinsker formulated their theoretical statement in a way that leads to the prediction of constancy for long-duration flashed backgrounds in our Condition D, it is important to deal further with the fact that this condition (and Condition $\mathrm{C}$ ) is not identical to the flashed background condition employed by Cornsweet and Pinsker. In their flashed $(4.5 \mathrm{msec})$ background condition, the increment was applied to the entire field of one of the two simultaneously flashed targets. Thus, their subjects were required to discriminate which of two nearby targets was brighter. In our experiment, the subject was required to detect an inhomogeneity in one of two targets. In the latter case, a comparison between the two targets was necessary only on trials when both targets or neither would appear to contain the inhomogeneity. Even so, the discriminable property of the configuration on which reporting was required was hardly a "relational" property between the two targets. In the Cornsweet and Pinsker case, a comparison between the two targets was an essential and intrinsic aspect of the task and could be "avoided" only if the subject retained some internal standard from previous trials and separately compared each of the two targets on a given trial to this standard; since it was so much easier-and undoubtedly more accurate and precise-to compare the two simultaneously compared targets to each other directly on a given trial, it is very likely that he did so. (This distinction may be made clearer by noting that in the present experiment the report of detection of the increment against one of the two backgrounds presented on a trial could be given even if the second background had not been presented, but in the Cornsweet and Pinsker experiment a report would be impossible if the second background were not also present.)

Whittle and Swanston (1974) have suggested that the above distinction is a fundamental one. In experiments with the Cornsweet and Pinsker paradigm, 
which appeared after the present article was accepted for publication, they reported that $\Delta \mathrm{L} / \mathrm{L}$ remained constant with $40-\mathrm{msec}$ flashes when both flashed targets were presented against darkness or against an $11^{\circ}, 3.05$ troland steady background. They suggest that the case in which $\Delta \mathrm{L} / \mathrm{L}$ decreases with increase in $\mathrm{L}$ involves "detection of a transient, that is, an edge in space or a brightness change in time or both at once," and that when $\Delta \mathrm{L} / \mathrm{L}$ is essentially constant with variation in $L$ a "discrimination between two transients" is involved. But our flashedbackground condition shows both forms-displaying constancy of $\Delta \mathrm{L} / \mathrm{L}$ for short flash durations and a monotonic decrease of $\Delta \mathrm{L} / \mathrm{L}$ for long flash durations. Thus, this distinction cannot provide the sole basis for determining the shape of the $\Delta \mathrm{L} / \mathrm{L}$ function unless we hold that our experiment involved detection of a transient for the brief flash and a discrimination between transients for the longer flash, a distinction for which there does not appear to be any basis.'

While further research is indicated, the following development of Cornsweet and Pinsker's approach may be worth considering: In any situation-discrimination or detection-the threshold value for a signal must finally depend on the level of noise against which the presence or absence of the signal is evaluated. This suggests that the subject's criterion for detection or discrimination is a minimum value of some function of $M_{s} / \sigma_{n}$, where $M_{s}$ is the mean neural response to the signal to be evaluated and $\dot{a}_{n}$ is the variability of the neural response to the background. (Cornsweet and Pinsker's model places the mean neural response to the background in the denominator, and would only conform to this formulation if it was assumed that the standard deviation of the response to the background increased at the same rate as its mean level.) This approach suggests that when $\Delta \mathrm{L} / \mathrm{L}$ does not remain constant as $\mathrm{L}$ varies, it is a consequence of a variation of $M_{s}$ with $\mathrm{L}$ that is not identical to the variation of $o_{\mathrm{n}}$ with $\mathrm{L}$.

It would be desirable to make use of data from electrophysiology to evaluate this. However, although there is relevant supporting evidence from recordings of the maintained discharge of the retinal ganglion cell of the cat [the standard deviation of the pulse number distribution by both on-center and off-center units to a steady light does not change much with light level (it may even decrease), while the mean firing level increases gradually for on-center units and is either unchanged or decreases gradually for off-center units (Barlow \& Levick, 1969)], appropriate comparable data do not appear to be available for transient stimuli. The evidence from cat electrophysiology regarding different units selectively responsive to transient and sustained stimuli (Cleland, Dubin, \& Levick, 1971; Rodieck, 1973; Stone \& Fukada, 1974) also supports some speculation regarding a basic difference in pro- cessing increments against steady and against flashed background: Specifically, both flashed test targets and flashed backgrounds may be processed by transient units. Hence, any change in the stimulusresponse relation with $\mathrm{L}$ for such a unit would be identically reflected in the response to both stimuli and leave their relation unchanged, thus providing a basis for $\Delta \mathrm{L} / \mathrm{L}$ constancy under some conditions for flashed-backgrounds. Steady backgrounds might be processed by sustained units and undergo a different change with $L$, thus providing a basis for variation in $\Delta \mathrm{L} / \mathrm{L}$ with $\mathrm{L}$ under a broad range of conditions; this difference with variation in $\mathrm{L}$ between sustained and transient units could be a difference in either variation of latency (see Footnote 7 ) or in gain (see Footnote 2).

\section{REFERENCES}

BARLOW, H. B. Temporal and spatial summation in human vision at different background intensities. Journal of Physiology, 1958. $141,337-350$.

BaRLow, H. B., \& Levick, W. R. Changes in the maintained discharge with adaptation level in the cat retina. Joumal of Physiology, 1969, 202, 699-718.

Baumgardt, E., \& SMTth, S. W. Facilitation effect of background light on target detection: A test of theories of absolute threshold. Vision Research, 1965, 5, 299-312.

Cleland, B. G., Dubin, M. W., \& Levick, W. R. Sustained and transient neurones in the cat's retina and lateral geniculate nucleus. Journal of Physiology, 1971, 217, 473-496.

Cornsweet, T. N. Visual perception. New York: Academic Press, 1970.

Cornsweet, T. N., \& Pinsker, H. M. Luminance discrimination of brief flashes under various conditions of adaptation. Journal of Physiology, 1965, 176, 294-310.

GrahaM, C. H.; \& KEMP, E. H. Brightness discrimination as a function of the duration of the increment in intensity. Journal of General Physiology, 1938, 21, 635-650.

Matin, L. Binocular summation at the absolute threshold of peripheral vision. Journal of the Optical Society of America, 1962, 52, 1276-1286.

Matin, L. Critical duration, the differential threshold, critical flicker frequency, and visual adaptation: $A$ theoretical treatment. Journal of the Optical Society of America, 1968, 58, 404-415.

MATIN, L. , \& Kornheiser, A. Linked changes in spatial integration, size discrimination, and increment threshold with change in background diameter. Vision Research, 1976, 16, 847-860.

RoDIECK, R. W. The vertebrate retina: Principles of structure and function. San Francisco: Freeman, 1973.

STEWART, B. R. Temporal summation during dark adaptation. Journal of the Optical Society of America, 1972, 62, 449-457.

Stone, J., \& FukUda, Y. Properties of cat retinal ganglion cells: A comparison of W-cells with $X$ - and $Y$-cells. Journal of Neurophysiology, 1974, 37, 722-748.

Whittle, P., \& Swanston, M. T. Luminance discrimination of separated flashes: The effect of background luminance and the shape of the T.V.I. curves. Vision Research, 1974, 14, 713-719.

\section{NOTES}

1. The identification of the time window with classical views of the nature of critical duration is not crucial. Although the time window is applied to the neural response and critical duration to the stimulus, no confusion results here from failing to distinguish the two. 
2. The term "gain" can be employed to refer to the ratio between any measures of output and input to a system. In order to facilitate comparison between the two theories, we employ it here in ways which, for each theory, involves that measure of neural response against which the subject's detection criterion is applied. Thus, while "gain" in Matin's theory refers to "instantaneous responsivity" (instantaneous neural response/stimulus intensity), in (Cornsweet and Pinsker's theory we employ "gain" to refer to responsivity as measured by the entire integrated neural response produced within a single time window (time integral of neural response/stimulus intensity). (We use "intensity" in the sense that Cornsweet (1970) has used the term in a delightful footnote (page 7) in his excellent book.]

3. Dr. Cornsweet has pointed out (personal communication) that his original intent in carrying out the experiments to which we refer was to measure increment thresholds under less complicated conditions than they are usually obtained: "Increment thresholds are usually measured with test flashes of relatively long duration against larger, steady backgrounds. Under these conditions, small eye movements cause unpredictable and ununderstood effects which interact with another set of ununderstood effects that happens at the border between the test flash and background. Although brief flashes on a dark background are certainly not simple, they are a lot simpler than long, unstabilized flashes and contours." We agree that small eye movements and lateral interaction acting separately and together at borders make the long-duration case more complicated, and for this reason will only be able to conclude that failure to find $\Delta \mathrm{L} / \mathrm{L}$ constancy with long-duration flashes argues against the sufficiency of Cornsweet's mechanism, but does not prove that it is not an operative mechanism.

4. Since a two-alternative spatial forced choice psychophysical method was employed, the probability of a correct response ranged from .5 to 1.0 under each condition. On the assumption that the distribution was normal in this range, the probabilities were linearly rescaled to a $0-1.0$ range so that the probit technique could be applied.

5. The dashed line in Figure 4 has a slope of 1.0. Hence, the magnitude by which the absolute threshold (as shown in the asterisked point) is larger than the $\Delta \mathrm{L}$ at the dimmest background is given by its height above the dashed line. Such facilitation by dim backgrounds has been reported before (Baumgardt \& Smith, 1965).

6. An interesting observation is also worth mentioning: On some trials, against each background the subject reported that flashes had occurred in both fields, that each was located in the center of its field, and that both were equally clear! There was no possibility of stimulus artifact. A similar phenomenon has been reported by Baumgardt and Smith (1965) in a multiple-interval forced choice paradigm. The fact that these flashes never appeared at inappropriate locations against the background in our observations suggesi that "emsory conditioning" or "atlentional" mechanisms (or both) are involved.

7. A difference in appearance between the 4 - and $200-\mathrm{msec}$ flash-background conditions is worth noting. In the $200-\mathrm{msec}$ condition, at the highest background luminance, the subjects reported that when the test flash was detected it often seemed to appear after the background had begun to fade in brightness. This is not surprising if we consider what is known about differences in response latency. It suggests, however, that at the highest background luminance the subject may have been detecting the test flash against the afterimage of the background and that the effect might be operative but less substantial at lower luminances. This could thus be a factor in the decrease in $\Delta \mathrm{L} / \mathrm{L}$ with $\mathrm{L}$ for the 200 -msec flashed background condition.

(Received for publication July 29, 1974; revision accepted August 5, 1976.) 